\title{
LIGHT SCATTERING AT NANO-TEXTURED SURFACES IN THIN FILM SILICON SOLAR CELLS
}

\author{
F.-J. Haug ${ }^{1}$, C. Battaglia ${ }^{1}$, D. Dominé ${ }^{2}$, C. Ballif ${ }^{1}$ \\ ${ }^{1}$ École Polytechnique Fédérale de Lausanne (EPFL), Institute of Microengineering (IMT), Photovoltaics and Thin Film \\ Electronics Laboratory, Rue A.-L. Breguet 2, CH-2000 Neuchâtel, Switzerland \\ ${ }^{2}$ now at University of Applied Sciences and Arts of Southern Switzerland (SUPSI), Institute for Applied Sustainability to \\ the Built Environment (ISAAC), $\mathrm{CH}-6952$ Canobbio
}

\begin{abstract}
State-of-the-art solar cells based on thin film silicon make use of random textures for absorption enhancement; in the past, the development of these textures was carried out empirically, and in most applications this is still the case. Attempts to understand light scattering at the internal interfaces rely on quantities like haze and angle resolved scattering that are only measurable in air and need to be extrapolated, normally by means of scalar scattering theory. In this context it is unfortunate that the description of scattering into air requires modifications with empiric parameters whose scaling is unknown. We present an alternate approach for predicting angular properties and intensities of scattered light which is based only on measurable quantities like the surface morphology and the refractive index dispersion, no adjustable parameters are needed.
\end{abstract}

\section{INTRODUCTION}

In thin film silicon solar cells the thickness of the photoactive silicon layer is normally much less than the absorption length, either to ensure an improved stability against light induced degradation in amorphous silicon, or because of throughput considerations in the case of microcrystalline cells. Thus, both types of thin film silicon solar cells require mechanisms for absorption enhancement by light scattering at internal interfaces. In the past, various types of interface texture have been developed empirically, but their performance in terms of absorption length enhancement still lacks significantly behind the theoretical prediction of $4 n^{2}$ where $n$ is the refractive index of silicon [1].

Despite impressive progress in rigorous calculation methods [2, 3], the current understanding of light scattering still relies largely on approximate descriptions and semi-empirical models [4-6]. The most widely used among those is scalar scattering theory which is valid when the wavelength of the incident radiation is larger than the typical feature size of the rough surface, generally represented by the root mean square roughness $\sigma_{\text {rms }}$. For the case of transmission, the dependence of the haze on the wavelength $\lambda$ is defined as the ratio of diffusely scattered to total light intensity [7].

$$
H_{T}=T_{\text {diff }} / T_{\text {tot }}=1-\exp \left\{-\left[(2 \pi / \lambda) \cdot \sigma_{\text {rms }} \cdot\left|n_{1}-n_{2}\right|\right]^{2}\right\}
$$

Here, $n_{1}$ is the refractive index of the incident medium, $n_{2}$ is the refractive index of the medium into which the scattered light propagates and $\sigma_{\mathrm{rms}}$ is the root mean square deviation of the profile with respect to its mean level. It can be measured, e.g. by atomic force microscopy (AFM). Based on measurements of the transmission haze into air, it was suggested to replace $\sigma_{\mathrm{rms}}$ in eq. (1) by a smaller effective roughness $\sigma_{\text {eff, }}$ and to change the exponent from its theoretical value of two into a freely adjustable parameter $\delta$ which is close to three for transparent conducting oxides (TCOs) with surface texture like $\mathrm{SnO}_{2}$ [5] or $\mathrm{ZnO}$ [8].

$$
H_{T} \approx T_{\text {diff }} / T_{\text {tot }}=1-\exp \left\{-\left[(2 \pi / \lambda) \cdot \sigma_{\text {eff }} \cdot\left|n_{1}-n_{2}\right|\right]^{\delta}\right\}
$$

In the actual device, scattering is from the TCO into silicon. Thus, model parameters adjusted to scattering measurements into air are not necessarily applicable.

In this contribution we present an alternate calculation for predicting angular properties and intensity of scattered light that does note require adjustable parameters [9], and we compare the results to experimental data of textured $\mathrm{ZnO}$ surfaces.

\section{MODEL}

Figure 1 depicts the light scattering situation in the way that is normally used in Fourier optics [10]. A plane wave coming from the left is incident on the rough surface described by $z=\zeta(x, y)$ and scattered into the half-space towards the right. The observer is assumed to sweep out the angle $\theta$ on a hemispherical observation screen. Scattering centres are described by an aperture function $U_{0}$; typical choices are the amplitude or the phase modulation. In case of amplitude modulation, $U_{0}$ is proportional to $\zeta(x, y)$ which yields a Fourier transformation of the height information. Eventually, the light intensity that is scattered into a given direction can be identified with a relation called power spectral density or surface factor $g\left(k_{x}, k_{y}\right)$ which contains the absolute square of the Fourier components [11-13]. From looking at a simple structure like a sinusoidal grating we conclude that a description with amplitude modulation not appropriate because the specular intensity would depend on the mean level of the surface, i.e. the layer thickness, and it yields only two nonspecular contributions. In the experiment, the specular intensity does depend on the grating amplitude, but it is 
little sensitive to the thickness of the substrate. Also, the prediction of two diffracted modes cannot account for the appearance of second orders, once the wavelength becomes less than half of the grating period. We still included PSD data in figure 5 because it is widely used, but it should be noted that the evaluation of the haze requires eqns. (1) or (2) because the normalization procedure outlined in the next section cannot be applied.

In the following we show that surface roughness is more adequately described as phase changing element. Before entering the roughness zone that is illustrated by the two vertical lines in Figure 1, the phase of the incident plane wave is given by $\varphi_{0}$. Within the roughness zone, the wave is approximated to propagate as plane wave, first for a distance $z_{1}$ in the medium with refractive index $n_{1}$, then the remaining distance $z_{2}$ in $n_{2}$. Interference effects are considered only after leaving the roughness zone on the way to the observer. The path across the roughness zone which is illustrated by the dashed line in fig (1) is thus replaced by a phase changing screen of zero thickness.

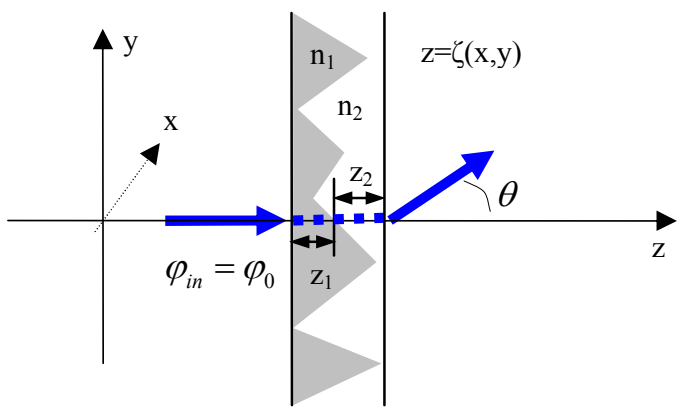

Figure 1: Definition of the phase difference during the transition of the roughness zone.

In order to calculate the phase change that is accumulated within the roughness zone, the optical path lengths in the two different materials are considered:

$$
\begin{aligned}
\varphi_{\text {out }} & =\varphi_{0}+\frac{2 \pi}{\lambda} n_{1} z_{1}(x, y)+\frac{2 \pi}{\lambda} n_{2} z_{2}(x, y) \\
& =\text { const }+\frac{2 \pi}{\lambda} \zeta(x, y)\left(n_{1}-n_{2}\right)
\end{aligned}
$$

The aperture function of the phase changing element can thus be described by $U_{0}=\exp \left\{\mathrm{i} \varphi_{\text {out }}\right\}$. The thickness of the substrate is contained in the constant; in accordance with observation it cancels when taking the absolute square.

Fourier optics distinguishes various approximations for the calculation of the scattered field amplitude in the right half space. Using a hemispherical observation screen and Fraunhofer approximation, the far field amplitude is obtained by a simple Fourier transform of $U_{0}$. The more accurate Fresnel approximation requires the evaluation of additional phase factors which can be related to well known aberration phenomena [14]. We did not incorporate these corrections here. It was shown by Harvey that a scaling of the input coordinates by the wavelength $\lambda$ (i.e. $\hat{x}=x / \lambda, \hat{y}=y / \lambda)$ relates the scattered amplitude distribution to the radiance $L$ which is then a function of the direction cosines $\alpha$ and $\beta$ [14] (see the appendix for more information on direction cosines).

$L(\alpha, \beta)=\frac{\lambda^{2}}{A_{s}}\left|\mathcal{F}\left\{U_{0}(\hat{x}, \hat{y})\right\}\right|^{2}$

Note that the radiance function is not a measurable intensity because it is defined for propagating and evanescent modes alike. The latter are characterized by values of $\alpha$ and $\beta$ outside the unit circle, i.e. beyond the horizon in a real observation space. In order to obtain measurable intensities, a normalization scheme must be applied where the transmitted energy is distributed only between those modes that are actually propagating [15]. This is achieved by defining the sum over all contributions within the unit circle $\alpha^{2}+\beta^{2}<1$ as total transmitted intensity $T_{t o t}$, stating essentially that evanescent modes outside the unit circle cannot be excited. We obtain a normalized radiance $L^{\prime}$ :

$$
L^{\prime}(\alpha, \beta)=\left\{\begin{array}{cl}
\frac{L(\alpha, \beta)}{\sum_{\alpha^{2}+\beta^{2}<1} L(\alpha, \beta)} & , \alpha^{2}+\beta^{2}<1 \\
0 & , \text { else }
\end{array}\right.
$$

Once the normalization is carried out, we can define experimentally accessible quantities. In order to relate $L^{\prime}$ to the intensity that is measured by a detector with fixed aperture size on the hemispherical observation screen, the projection of the emitting surface that is actually visible to the detector must be taken into account by a factor of $\cos \theta$ [15]. This yields a general definition for a two dimensional angle resolved scattering $\operatorname{ARS}_{2 \mathrm{D}}(\alpha, \beta)$.

For random surface textures and perpendicular incidence it is sufficient to evaluate a one-dimensional ARS. It corresponds to a measurement where the detector does not sweep the full hemisphere but only a meridian that contains the $z$-axis. Thus, the ARS can be extracted from the two dimensional distribution either by evaluating one coordinate only, or by carrying out an average over the azimuthal angle $\psi$ around the $z$-axis. We chose the latter procedure because it reduces noise in the data.

Rather than looking at the complete angular information at each individual wavelength, sometimes only the spectral haze is desired. Because the specular transmission $T_{\text {spec }}$ upon perpendicular incidence is identified with the contribution of the radiance at $\alpha=\beta=0$, eqns. (1) or (2) are no longer required; haze in transmission can be defined by:

$$
H_{T}=\frac{T_{\text {diff }}}{T_{\text {tot }}}=\frac{T_{\text {tot }}-T_{\text {spec }}}{T_{\text {tot }}}=1-\frac{T_{\text {spec }}}{T_{\text {tot }}}=1-L^{\prime}(0,0)
$$




\section{EXPERIMENTAL}

We investigate random textures of zinc oxide which is used as transparent conducting substrate for thin film silicon solar cells in $p-i-n$ configuration. The films are grown by low pressure chemical vapour deposition (LPCVD) on glass substrates (Schott, AF45). We discuss two types of $\mathrm{ZnO}$ films called $\mathrm{Z2}$ and $\mathrm{Z} 5$ which refers to their approximate thicknesses in micrometers. For certain choices of growth conditions, LP-CVD ZnO develops a surface texture that consists of pyramidal facets [16]. The size of these features scales with the thickness, the finegrained $Z 2$ and the coarse-grained $Z 5$ films are adapted to amorphous and microcrystalline cells, respectively. A constant sheet resistance of $10 \Omega \square$ is maintained in both types of $\mathrm{ZnO}$ by adjusting the boron doping [17].

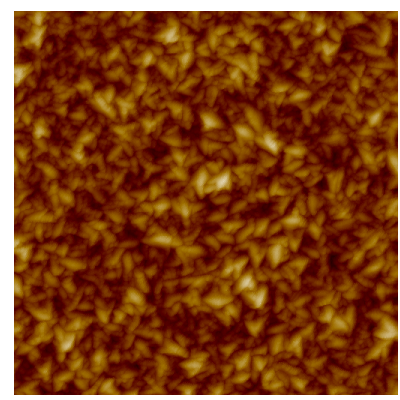

Z2
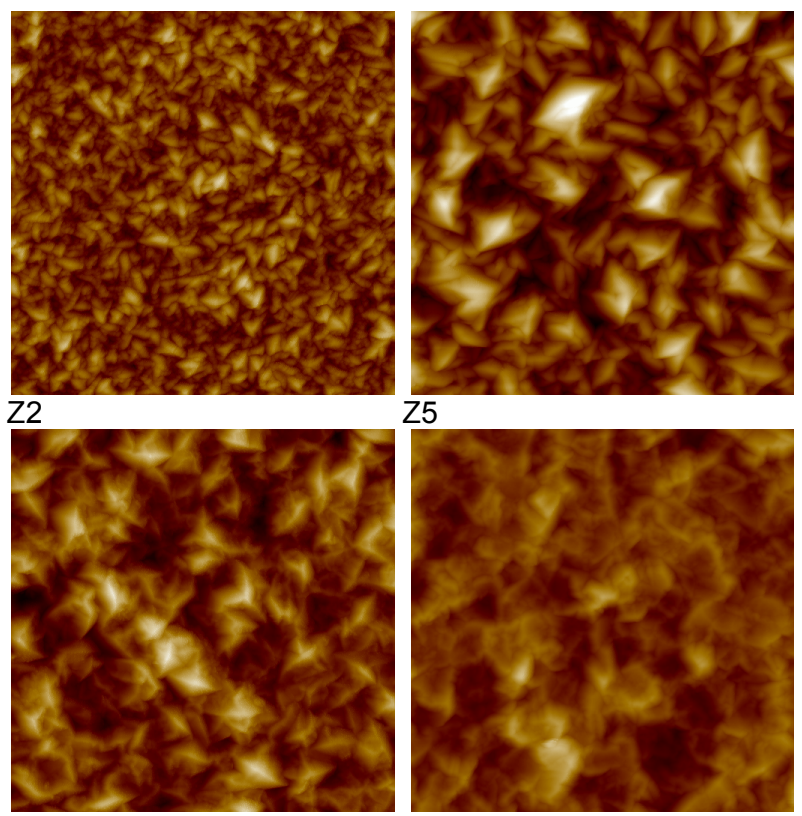

Z5

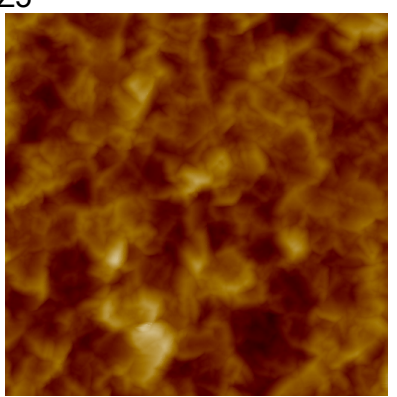

Z5(20 min)

Z5(60 min)

Figure2: AFM surface morphologies of the textured $\mathrm{ZnO}$ substrates used in this investigation (image size: $10 \times 10{\mu m^{2}}^{2}$ )

The weaker doping in the $Z 5$ films also reduces the free carrier absorption in the near IR which is advantageous for better response at long wavelengths in microcrystalline solar cells. However, Z5 films require an additional plasma treatment that converts $\mathrm{V}$-shaped troughs between the facets into round U-shaped valley; the treatment slightly reduces the absorption enhancement that the texture can achieve in the cell, but it yields more favourable performance in terms of open circuit voltage and fill factor [18].

The haze of the $\mathrm{ZnO}$ films is measured in the spectral range between 400 and $2000 \mathrm{~nm}$ in a spectrophotometer with integrating sphere (lambda 900, Perkin Elmer), the ARS is determined with laser illumination at $543 \mathrm{~nm}$. In both cases, the incidence is from the glass side. Refractive indices of the $\mathrm{ZnO}$ films are determined with spectroscopic ellipsometry (Horiba), the surface profiles are measured by AFM; areas of $10 \times 10 \mu \mathrm{m}^{2}$ are probed with a resolution of $512 \times 512$ pixels (DI 3100 , Digital Instruments).

\section{RESULTS}

The AFM surface morphologies of the four textures are shown in figure 2 . The pyramidal features of $Z 2$ and Z5 are similar in shape, but differ in size. The values of the rms roughness are 70 and $180 \mathrm{~nm}$ for the $Z 2$ and the $Z 5$ textures, respectively. The plasma treatment does not significantly change the thickness, but after durations of 20 and 60 minutes the rms roughness is reduced to 150 and $120 \mathrm{~nm}$, respectively.

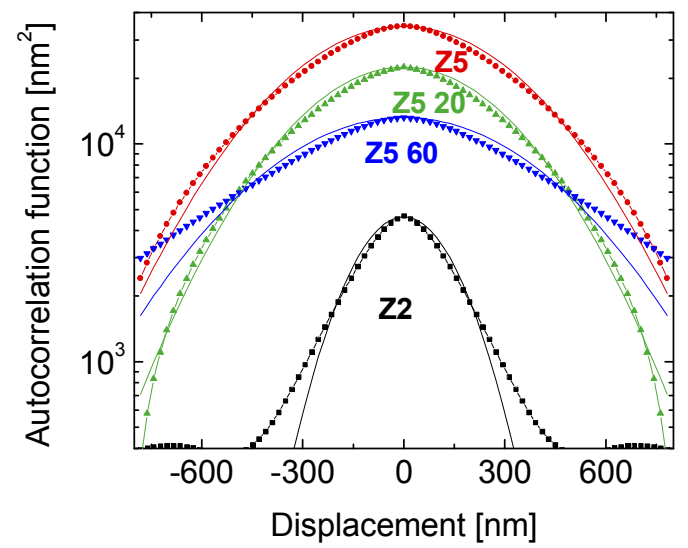

Figure 3: Plots of the auto-correlation length for the different zinc oxide textures (symbols) and the respective Gaussian approximations (lines).

The scalar scattering theory underlying to eq. (1) is based on a height profile where Gaussian distributions are assumed for the height deviation from the surface mean, as well for the autocorrelation function.

$A C F=\sigma_{r m s}^{2} \cdot \exp \left\{-\left(x^{2}+y^{2}\right) / \Lambda^{2}\right\}$

Eq. (7) involves two quantities, the root mean square roughness $\sigma_{\mathrm{rms}}$ and the correlation length $\Lambda$, although the latter does not appear in eq. (1) because it gets cancelled in the approximations that lead to it. It is still instructive to study the autocorrelation function of the experimental AFM data. As expected from the random nature of the texture, the 2D autocorrelation functions do not show significant azimuthal variations; it is thus sufficient to plot onedimensional cuts as shown in figure 3 . From the radii where the functions drop to $1 / \mathrm{e} \approx 0.37$ of their respective maxima, we estimate correlation lengths of $200 \mathrm{~nm}$ and $470 \mathrm{~nm}$ for $\mathrm{Z2}$ and Z5, respectively. With the plasma treatment $\Lambda$ first narrows to $420 \mathrm{~nm}$ on the $20 \mathrm{~min}$ sample, 
but then widens to $540 \mathrm{~nm}$ on the $60 \mathrm{~min}$ sample. In general the Gaussian of eq (7) is a sufficiently good approximation around the maxima, but there are deviations for large displacements, particularly so for the 60 min treated $Z 5$.

The spectral haze upon transmission through the four different surface textures is shown in figure 4 . The figure includes predictions of scalar scattering theory according to eqns. (1) and (2). We projected two different scenarios for the exponent $\delta$, setting it equal to two as in the original theory, and changing it to a value of three as proposed in the literature $[5,8]$. For better comparability, we did not change the roughness but used $\sigma_{\text {rms }}$ of the AFM analysis in both cases. It is observed that neither model is valid in a wide spectral range, but it appears that a choice of $\delta$ equal to two is better when the haze values are above $60 \%$ while $\delta$ equal to three fits better below $50 \%$. This is, however, a mere observation rather than a physically justified criterion. It is likely limited to the shown type of textures and scattering into air, there is no way to predict the value of $\delta$, nor how it will behave for other materials.

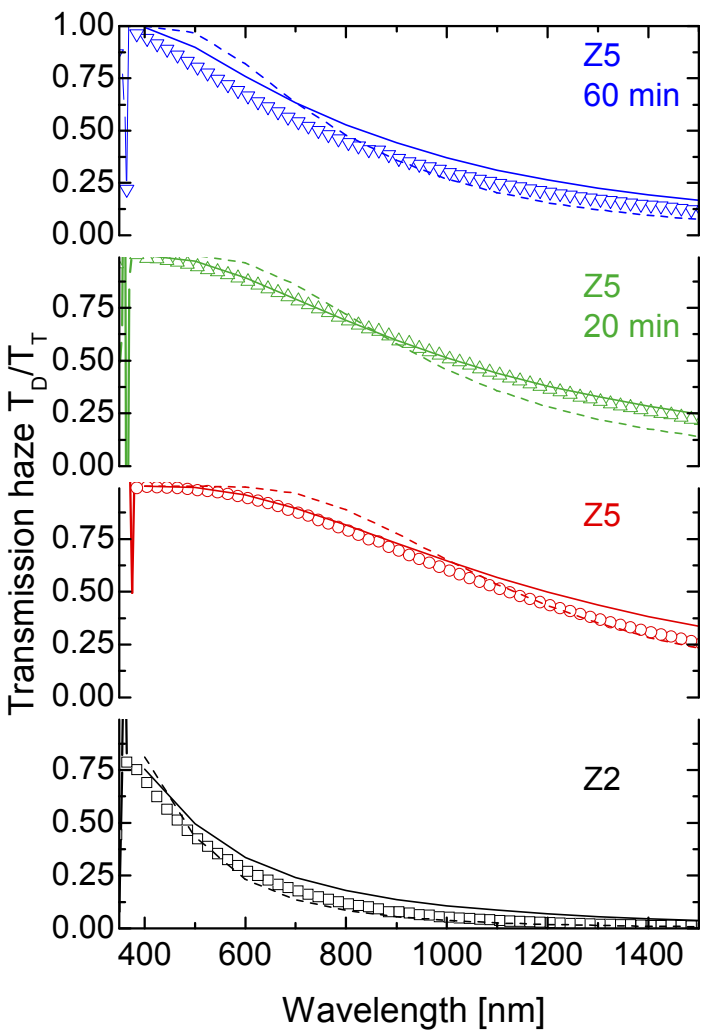

Figure 4: Spectral haze of the four surface textures (symbols). Full lines represent predictions according to eq. 1 , dashed lines are according to eq. 2 , using $\delta$ equal to three.

Figure 5 shows ARS characteristics of the four surfaces. The scale applies to results obtained from the normalized radiance model (thick full lines); integrating between zero and $90^{\circ}$ yields unity if carried out in 2D polar coordinates and units of radian. The experimental curves have been scaled by a common factor that takes into account aperture size and sensitivity of the detector. Dashed lines illustrate the theoretical concept of a Lambertian which scatters the same intensity into all angles. Within the terminology used here, this means constant radiance but an ARS which is proportional to $\cos \theta$.

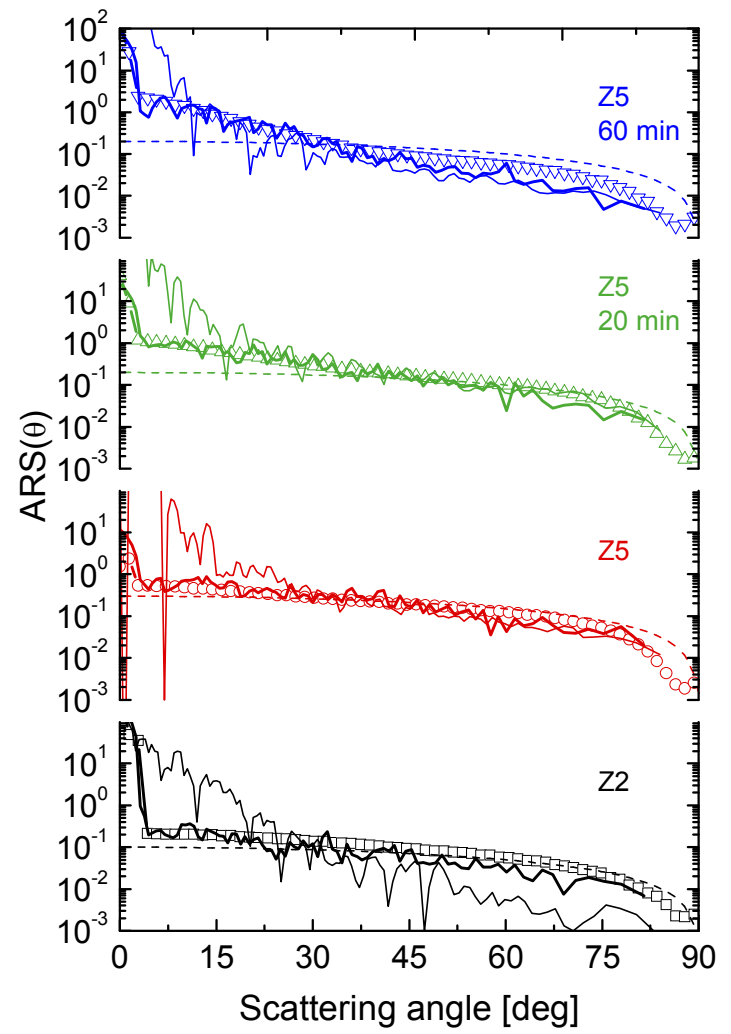

Figure 5: Angle resolved scattering of the four textures at $543 \mathrm{~nm}$ (symbols). Thin and thick full lines show results of Fourier approach using amplitude and phase modulation, respectively, dashed lines illustrate Lambertian scattering.

All measured curves in figure 5 show specular transmission at low angles. At high angles there is a decrease due to the projection factor of $\cos \theta$ which is inherent to the measurement. Scattering of the $\mathbf{Z 2}$ and the $\mathbf{Z} 5$ textures exhibits a broad angular range, similar to Lambertian scattering. The $\mathrm{Z} 5$ texture shows a smaller contribution of the specular beam and scattered intensities that are higher by a factor of about three to four compared to the Z2 texture. The samples with plasma treatment exhibit preferential scattering into lower angles. We observe an excellent agreement between the experimental characteristics and the curves calculated with the phase change model. Besides the issue with the specular beam, the PSD model tends to overestimate the contributions at 
small angles, presumably because it cannot predict scattering into higher orders as discussed above.

The haze could be accessed from the shown curves by separating specular and diffuse parts, but we prefer to evaluate the haze directly according to eq. (6) because the ARS characteristics are only obtained after interpolation and noise reduction.

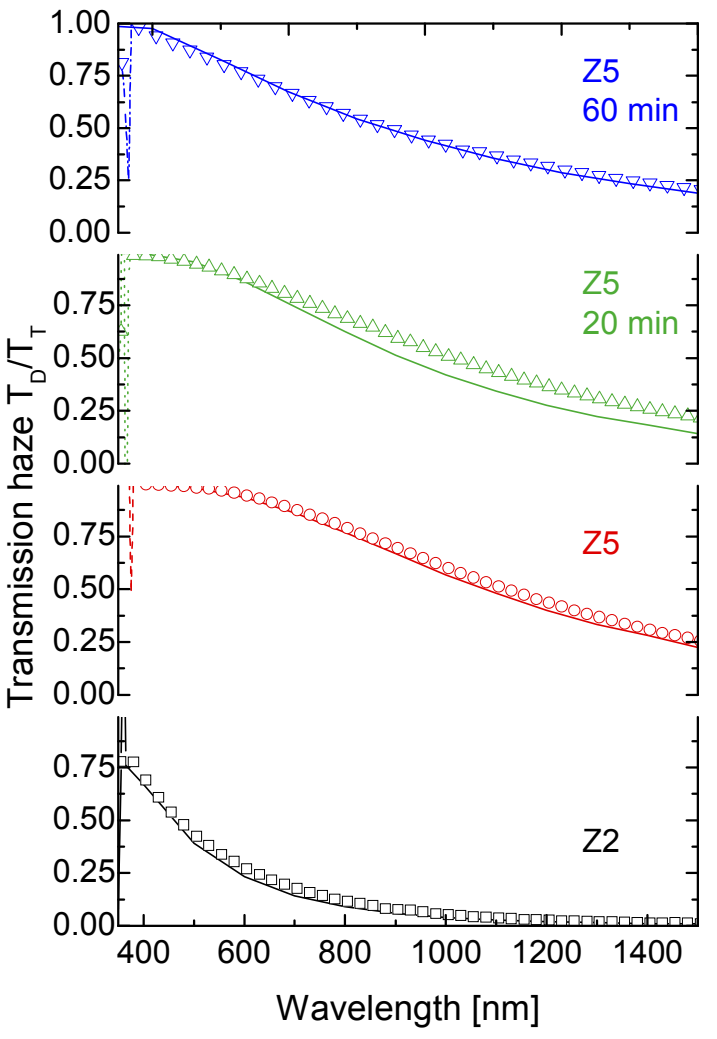

Figure 6: Comparison of measured haze characteristics (symbols) and scattering according to the Fourier approach (lines).

Figure 6 presents a comparison of the measured spectral haze with calculated characteristics using the Fourier approach. Except for the $20 \mathrm{~min}$ treated sample, there is an excellent correspondence over the full range of wavelengths. At this time we cannot explain why this particular surface deviates while the untreated as well as more strongly treated Z5 textures are described adequately. Looking at the ARS curves of figure 5 does not hint at any particular deviations, neither. Even with the exception, it can still be concluded that the prediction of light scattering by the Fourier model represents a huge improvement over the scalar theory of eqns. (1) or (2) because achieves an excellent description without any adjustable parameters. Therefore, the model also allows a prediction of the actual light scattering that takes place at the interface between $\mathrm{ZnO}$ and silicon in an actual device.

\section{DISCUSSION}

The interpretation of surface roughness as phase changing element yields a significantly improved description of light scattering, not only compared to statistic models of scalar scattering theory, but also over formulation in terms of the PSD which in fact represents a treatment with an amplitude changing element. The presented approach yields a quantitative two-dimensional distribution of the scattered light intensity, including the specular beam. From this function, the 1D-ARS and the haze are easily determined. Current non-rigorous models for the prediction of optical device performance are normally based on scalar scattering theory and follow a different approach [4-6]; they start by projecting the amount of specular and diffuse light for each wavelength. For a prediction of the haze, the parameters $\sigma_{\text {eff }}$ and $\delta$ are obtained by fitting eq. (2) to measured data in air. Then, these parameters are used to project the haze of a given interface in the device by inserting the respective refractive indices of the desired material combination into eq. (2). Finally, the propagation of specular and diffuse light is calculated separately, using coherent wave optics for the specular part and incoherent ray optics for the diffuse part. Further assumptions must be made for the angular distribution of the diffuse part; either appropriate functions like Lambertian scattering are assumed, or ARS data from a measurement in air is used. Based on this procedure, an absorption profile is calculated.

Avoiding the uncertainties that are associated wit the use of scalar scattering theory is expected to significantly improve the predictive power of device modeling. We propose to use the presented Fourier model for this purpose because it provides a consistent set of haze and ARS at little extra cost. Compared to scalar theory, the only additional requirement is the surface topography, but AFM measurements should be accessible in most research labs. In terms of computational effort, there is only the Fourier transform of the aperture function which can be handled easily by the FFT algorithm within fractions of a second. The subsequent evaluation of ARS and haze consists of simple manipulations like sums and the scaling of row and column indices.

\section{CONCLUSIONS}

We presented a Fourier approach for the description of light scattering at the interface texture between two dielectric materials. The model can quantitatively predict the angular properties as well as the intensity of the scattered light in transmission. The model is validated with measured data of haze and angle resolved scattering from textured $\mathrm{ZnO}$ surfaces. We find that the Fourier model is a clear improvement over scalar scattering theory because it is capable of correctly describing ARS and haze in a wide spectral range. This is remarkable because the model requires only the topography of the texture and the refractive indices of the adjacent materials, but it does not contain any adjustable parameters whatsoever. 


\section{APPENDIX}

The direction cosines $\alpha$ and $\beta$ are defined by $\alpha=x / r$ and $\beta=y / r$ where $r$ is the distance from the origin to the observer. They are normally defined with respect to the elevation above the equatorial plane because they project the normalized radius vector into that plane. Sticking to the polar angle $\theta$ as defined in figure 1 , we obtain $\alpha=\sin \theta$ $\cos \psi$ and $\beta=\sin \theta \sin \psi$ (with respect to the surface normal they are "direction sines"; but the name "direction cosine" better expresses aspect of projection into the plane). As all shown results are rotationally invariant around the $z$-axis, it is sufficient to consider $\alpha=\sin \theta$ in the $x-z$ plane. In direction cosine space, Snell's law of refraction at the interface between two dielectrics with refractive indices $n_{1}$ and $n_{2}$ is conveniently expressed as:

$$
n_{1} \alpha_{1}=n_{2} \alpha_{2}
$$

The grating equation becomes equally compact:

$$
n_{1} \alpha_{1}-n_{2} \alpha_{2}=m \frac{\lambda}{\Lambda}
$$

Forbidden grating orders are distinguished by direction cosines greater than one, this was already used for the normalization of the radiance in eq. (5).

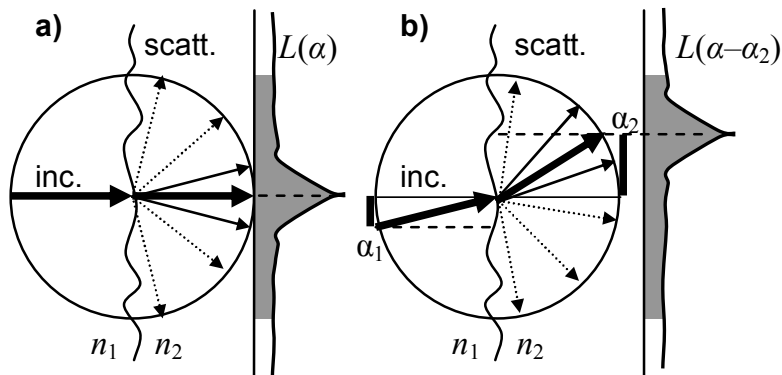

Figure A1: Diagram a) illustrates perpendicular incidence and the radiance after scattering in terms of direction cosines. Linear shift of the radiance upon oblique incidence is depicted in b). Shaded areas represent the normalization into propagating modes $\left|\alpha_{2}\right|<1$.

The concept of direction cosines becomes particularly useful when dealing with the radiance $L$. When the illumination is incident under an angle $\theta_{1}$ (corresponding to a direction cosine of $\alpha_{1}$ in medium 1), the incident phase depends linearly on the $x$-coordinate. Applying the shift theorem of the Fourier transform then yields linear shift of the complete radiance function $L$ by an amount of $\alpha_{2}$ [15]. Normalization into $L^{\prime}$ and conversion into measurable quantities is carried out subsequently.

\section{ACKNOWLEDGEMENTS}

Support from the Swiss National Office for Energy (OFEN) under project No. 101191 is thankfully acknowledged.

\section{REFERENCES}

[1] E. Yablonovitch and G.D. Cody, "Intensity enhancement in textured optical sheets for solar cells", Electron Devices, IEEE Transactions on, 1982. 29(2), p. 300-305.

[2] C. Haase and H. Stiebig, "Thin-film silicon solar cells with efficient periodic light trapping texture", Applied Physics Letters, 2007.91(6), p. 061116

[3] C. Rockstuhl, et al., "Local versus global absorption in thin-film solar cells with randomly textured surfaces", Applied Physics Letters, 2008. 93, p. 061105.

[4] J. Krc, F. Smole, and M. Topic, "Analysis of light scattering in a-Si:H solar cells by a one-dimensional semicoherent optical model", Progress in Photovoltaics, 2003. 11(1), p. 15-26.

[5] M. Zeman, et al., "Optical modeling of a-Si:H solar cells with rough interfaces: Effect of back contact and interface roughness", Journal of Applied Physics, 2000. 88, p. 6436. [6] J. Springer, A. Poruba, and M. Vanecek, "Improved three-dimensional optical model for thin-film silicon solar cells", Journal of Applied Physics, 2004. 96, p. 5329.

[7] C.K. Carniglia, "Scalar scattering theory for multilayer optical coatings", Optical Engineering, 1979. 18(2), p. 104-115.

[8] H. Stiebig, et al. "Light scattering in microcrystalline silicon thin film solar cells", in Proc. 16th EU-PVSEC. 2000. Glasgow, p. 549-552.

[9] D. Dominé, et al., "Modeling of light scattering from micro- and nanotextured srufaces", Journal of Applied Physics, 2010. 107, p. 044504-8.

[10] J. Goodman, Introduction to Fourier optics. 2005: Roberts \& Company Publishers.

[11] V. Celli, A. Marvin, and F. Toigo, "Light scattering from rough surfaces", Physical Review B, 1975. 11(4), p. 1779-1786.

[12] J. Elson and J. Bennett, "Relation between the angular dependence of scattering and the statistical properties of optical surfaces", Journal of the Optical Society of America, 1979. 69(1), p. 31-47.

[13] J. Elson and J. Bennett, "Calculation of the power spectral density from surface profile data", Applied Optics, 1995. 34(1), p. 201-208.

[14] J. Harvey, "Fourier treatment of near-field scalar diffraction theory", American Journal of Physics, 1979. 47, p. 974.

[15] J. Harvey, et al., "Diffracted radiance: a fundamental quantity in nonparaxial scalar diffraction theory", Applied Optics, 1999. 38, p. 6469-6481.

[16] S. Faÿ, et al., "Opto-electronic properties of rough LPCVD ZnO:B for use as TCO in thin-film silicon solar cells", Thin Solid Films, 2007. 515(24), p. 8558-8561.

[17] J. Steinhauser, et al. "Effect of rough $\mathrm{ZnO}$ layers in improving performances of microcrystalline silicon solar cells", in Proc. 20th European PVSEC. 2005. Barcelona, p. 1608-1611.

[18] J. Bailat, et al. "High efficiency pin microcrystalline and micromorph thin film silicon solar cells deposited on LPCVD ZnO coated glass substrates", in Proc. 4th World PVSEC. 2006. Hawaii, p. 1533-1536. 